\title{
REVISTA DE REVISTES
}

\author{
A cura d'Antoni Estradé \\ (Universitat Autònoma de Barcelona)
}

La selecció d'articles que tot seguit oferim ha estat feta a partir de les revistes de ciències socials corresponents a l'any 1982 de què hom disposa a la Biblioteca de Ia Facultat de Ciències Econòmiques i Empresarials de la Universitat Autònoma de Barcelona, arribades durant l'any 1982 i fins al 10 de gener de 1983. Procedim a esmentar-les per ordre alfabètic:

«Actes de la Recherche en Sciences Sociales» (ARSS)

«American Journal of Sociology» (AJS)

«American Sociological Review» (ARS)

«Archives Européennes de Sociologie» (AES)

«L'Avenç" (L'A)

"The British Journal of Sociology» (BJS)

«Cahiers Internationaux de Sociologie» (CIS)

«Ciencias Sociales» (CiSo)

"Communications» (C)

"Current Sociology» (CS)

«Economy and Society» (E\&S)

«Espaces et Sociétés» (ES)

«Government and Opposition» (GO)

«The Insurgent Sociologist» (IS)

«Journal of the History of Ideas» (JHI)

«The Journal of Peasant Studies» (JPS)

«Journal for the Scientific Study of Religion» (JSSR)

«Monthly Review» (MR) 
«Papers»: Revista de Sociologia

«New Left Review» (NLR)

«The New Hungarian Quartely» (NHQ)

«Past and Present» (P\&P)

«La Pensée» (LP)

«Population» (P)

«Problemi del Socialismo» (PS)

"Quaderni Rassegna Sindacale» (QRS)

«The Review of Radical Political Economics» (RRPE)

«Revista de Estudios Agro-Sociales» (REAS)

«Revista de Estudios Políticos» (REP)

«Revista Española de Investigaciones Sociológicas» (REIS)

«Revue Française de Sociologie» (RFS)

«Social Compass» (SC)

«Sociologia» $(\mathrm{S})$

«Sociología del Trabajo» (ST)

"The Sociological Review» (SR)

\section{ECOLOGIA HUMANA I DEMOGRAFIA}

Jean Baechler. La nourriture des bommes. Essai sur le néolitbique, AES 2, 1982: 241-293.

Daniel Courgeau et Mònique Lefebvre. Les migrations internes en France de 1954 à 1975. II. Migrations et urbanisation, $\mathrm{P}$, núm. 2, 1982: 341.370 .

Andràs Klinger. Population Policy in Hungary-Scope and Limits, NHQ, núm. 85, 1982: 115-124.

Amando de Miguel. La población en Madrid en los primeros años del siglo, REIS, núm. 19, 1982: 55-71.

Michèle Tribalat. Cbronique de l'Immigration, P, núm. 1, 1982: 131-157.

\section{HISTORIA, TEORIA I METODOLOGIA}

Georges Balandier. La sociologie aujourd'bui, CIS, núm. S, 1981: 197-204. Helena Béjar. Rousseau: opinión pública y voluntad general, REIS, número 18,1982 : $69-82$.

Maura de Bemart. I documenti personali. Metodologia americana nelle scienze sociali 1938-1945, S, núm. 1, 1982: 67-97.

John Bossy. Some Elementary Forms of Durkheim, P\&P, num. 95, 1982: 3-18. 
Ftançois Bourricaud. Contre le prophetisme en sociologie, CIS, núm. S, 1981: 227-242.

Jordi de Cambra Bassols. La teoría critica y el problema del método en las ciencias sociales, REIS, núm. 17, 1982: 53.64.

Colin Campbell. Merton's Concepts of Manifest and Latent Function, ASR, núm. 1, 1982: 29-44.

Renato Cavallaro. Individuo, gruppo, società: un confronto bra quattro «classici», S, núm. 1, 1982: 31-66.

Peter A. Corning. Durkbeim and Spencer, BJS, núm. 3, 1982: 359-382.

Michael Donnelly. Foucault's genealogy of the buman sciences, E\&S, nímero 4, 1982: 363-380.

Dong Dow. Marxism for the few, or, let'em Eat theory, MR, núm. 11, 1982: $14-29$.

Gilbert Durand: Le social et le mytbique. Pour une toptque sociologique, CIS, núm. S, 1981: 289-308.

Tony Fahey. Max Weber's «Ancient Judaism», AJS, núm. 1, 1982; 62-87.

Robert Golding. Freud, psychoanalysis, and sociology: some observations on the sociological analysis of the individual, BJS, num. 4, 1982: $545-562$.

Leslie F. Goldstein. Early Feminist Themes in French Utopian Socialism: The Saint-Simonians and Fourier, JHT, núm. 1, 1982: 91-108.

A. R. Halsey. Provincials and Professionals: the British Post-War Sociologists, AES, núm. 1, 1982: 150-175.

Donald Hodges and Ross Gandy. Marx and Economic Determinism, RRPE, núm. 1, 1982: 33-41.

Benjamin Kilbonne. Antbropological thought in the wake of the French Revolution: the Société des observateurs de l'bomme, AES, núm. 1, 1982: 73-91.

Reinhart Koselleck. Begriffsgescbichte and social bistory, E\&S, núm. 4, 1982: $409-427$.

David E. Leary. The Fate and Influence of Jobn Stutart Mill's Proposed Science of Etbology, JHI, núm. 1, 1982: 153-162.

Raymond Ledrut. Métbode ou méthodes, CIS, núm. S, 1981: 215-226.

Harry Liebersohn. Leopold pon Wise and the Ambivalence of Functionalist Sociology, AES, núm. 1, 1982: 123-149.

Carmelo Lisón Tolosana. La singularidad plural. Antropología cultural y especifidad, REIS, núm. 18, 1982: 7-27.

Rafael López Pintor y José Ignacio Wert Ortega. La otra España. Insolidaridad e intolerancia en la tradición político-cultural española, REIS, núm. 19, 1982: 7-25.

Josep Ramon Llobera. Wallerstein i l'antropologia del Mediterrani, L'A, nútn. $49,1982: 62-65$.

Michel Maffesoli. Pour une sociologie relativiste, CIS, núm. S, 1981: 205-214. 
«Papers»: Revista de Sociologia

J. Mandzhian. Apreciaciones alternativas al progreso bistórico, CS, núm. 2, 1982: 32.42 .

Pierre Manent. Tocqueville's Democratic Man, GO, núm. 2, 1982: 195-210.

Artemis March. Female Invisibility in Androcentric Sociological Theory, IS, núm. 2, 1982: 99-108.

Janina Markiewic-Lagneau. Florian Znaniecki, sociologue de l'action sociale et de la métbode analytique, RFS, núm. 2, 1982: 171-193.

John Martell. Teacbing the «Economic and Pbilosopbical Manuscripts of 1844»: Human-Centered Economics, RRPE, núm. 4, 1982: 53-62.

J. Martínez Alier and J. M. Natedo. A Marxist Precursor of Energy Economics: Podolinski, JPS, núm. 2, 1982: 207-224.

Eionel A. McKenzie. Rousseau's Debate with Macbiavelli in the Social «Contract», JHI, núm. 2, 1982: 209-228.

Abraham A. Moles. Pensée rigoureuse et sciences du vague: du bon usage des matbématiques dans les sciences sociales, CIS, núm. S, 1981: 269-288.

Christian de Montlibert. La professionalisation de la sociologie et ses $l i$. mites, RFS, núm. 1, 1982: 37-54.

Edgar Morin. Peut-on concevoir une science de l'autonomie?, CIS, núm. S, 1981: 257-268.

Gert H. Mueller. Socialism and Capitalism in the work of Max Weber, BJS, núm. 2, 1982: 151-171.

Richard Münch. Talcott Parsons and the Theory of Action. II. The Continuity of the Development, AJS, núm. 4, 1982: 771-826.

Bernat Muniesa. Capitalisme, societat i darwinisme, L'A, núm. 48, 1982: $32-34$.

Luis Núñez Ladeveze. Auguste Comte y la división del trabajo social, REP, núm. 26, 1982: 7-34.

Catherine Rhein. La géograpbie, discipline scolaire et/ou science sociale?, RFS, núm. 2, 1982: 223-251.

José M. Rodríguez Paniagua. Jobn Stuart Mill: su utilitarismo, su ética, su filosofia politica, REP, núm. 25, 1982: 7-24.

Edmund N. Santurri. Tbeodicy and Social Policy in Maltbus' Thought, JHI, núm. 2, 1982: 315.330.

Stephen A. Satris. The Theory of Value and the Rise of Etbical Emotivism, J.H.I., núm. 1, 1982: 109-128.

Mashe Shokeid. Toward an anthropological perspective of fairy tales, SR, núm. 2, 1982: 223.233.

Dennis Smith. Social bistory and sociology-more than just good friends?, SR, núm. 2, 1982: 286-308.

Sheelagh Strawbridge. Altbusser's theory of ideology and Durkheim's account of religion: an examination of some striking parallels, SR, núm. 1 , 1982: $125-140$.

André Tabouset-Keller. La conscience détrônée: de Freud a Lacan, LP, $229,1982$. 
Nancy di Tomaso, Sociological Reductionism from Parsons to Altbusser, ASR, núm. 1, 1982: 14-28.

Alain Touraine. Le retour de l'acteur, CIS, núm. S, 1981: 243-256.

Bryan S. Turner. Nietzsche, Weber and the Devaluation of Politics, SR, núm. 3, 1982: 367.391.

Luciano Verdone. La sociologia giuridica weberiana nella critica di Gurvitch, S, núm. 1, 1982: 99-122.

Gerard Vilar. Kant i nosaltres, L'A, núm. 47, 1982; 44-47.

Alan Warde. E. P. Thompson and 'poor' theory, BJS, núm. 2, 1982: 224-237.

Richard Wolff and Steven Resmick. Classes in Marxian Tbeory, RRPE, núm. 4, 1982: 1-18.

Geoffrey Wood. Frazer's Magic Wand of Antbropology: Interpreting'The Golden Bough, AES, núm. 1, 1982: 92-122.

\section{Monogràfics:}

Dossier: Roland Barthes, C, núm. 35, 1982.

Articles de: Edgar Morin, Umberto Eco, Jacques Le Goff, Julia Kristeva, François Wahl i Roland Barthes (entre d'altres).

Número monogràfic sobre Multi-level research. Article únic:

Pieter Von den Eeden and Harry J. M. Hüffnerr. Multi-level research, C.S., núm. 3, 1982: 1-117. Ve acompanyat d'una Bibliografia sobre el tema de 346 crèdits.

\section{ORGANITZACIO SOCIAL, SISTEMA SOCIAL,} ESTRUCTURA SOCIAL

Jacques Anuequin. Structures sociales et politiques dans l'Antiquité. Stratification et/ou étude des rapports sociaux, LP, núm. 225, 1982: 118-128.

Aaron V. Cicourel. Procedimientos interpretativos y reglas normativas en la negociación del status y rol, REIS, núm. 19, 1982: 13-104.

Francis Cohen. Structure sociale, classes et différenciations dans le socialisme existant (notes préliminaires), LP, 225, 1982: 91-102.

Félix Damette. Classes, luttes de classes, mouvement social, LP, 225, 1982: 55-63.

Robert Erikson, John H. Goldthorpe and Lucienne Portocavero. Social fluidity in industrial nations: England, France and Sweden, BJS, número 1, 1982: 1.34.

Richard L. Henshel. The boundary of the self-fulfilling prophecy and the dilemma of social prediction, BJS, núm. 4, 1982: 511-528. 
Keith Hope. Vertical and Non-Vertical Closs Mobility, ASR, 1982; 100-113. Jean Pierre Lefebvre. Marx: deux classes ou trois?, LP, 225, 1982: 77-90. Varda Leymore. The Structural factor in systems of communication, BJS, núm. 3, 1982: 421-434.

Michel Maffesoli. La Maffia. Note sur la socialité de base, CIS, núm. D, 1982: $363-368$.

Ramesh Mishta. System integration, social action and change: some problems in sociological analysis, SR, núm. 1, 1982: 5-22.

Eckart Pankoke. Social movement, E\&S, núm. 3, 1982: 317-346.

Ray Pawson. Desperate measures, BJS, núm. 1, 1982: 35-63.

Michel Pollak. Des mots qui tuent. ARSS, núm. 41, 1982: $29-45$.

Survivre dans un camp de concentration. Entretien avec Margareta GlasLarsson, commenté par Gerbard Botz et Michel Pollak, ARSS, núm. 41, 1982: $3-28$.

\section{SOCIOLOGIA DEL CONEIXEMENT I DE LA CULTURA}

Mahmoud Amin el Alem. Le dualisme «terre-ciel» dans la pensée arabomusulmane contemporaine, LP, núm. 229, 1982: 80-90.

Margaret S. Archet. Morpbogenesis versus structuration, BJS, núm. 4, 1982: $455-483$.

James A. Beckford. The Ideologies of Privacy, CS, núm. 2, 1982: 43-66. Miguel Beltrán. La realidad social como realidad y apariencia, REIS, número 19, 1982: 27.53.

Walter Benjamin. Goethe: The Reluctant Bourgeois, NLR, núm. 133, 1982: 69.93 .

Anthony Bryant. The Case of Raymond Williams, JS, núm. 2, 1982: 89-98.

Nicholas Canny. The formation of the irish Mind, Religion, politics and Galic Irish Literature 1580-1750, P\&P, núm. 95, 1982: 91-116.

James G. Carrier. Knowledge, Meaning and Social Inequality in Kennetb Burke, AJS, núm. 1, 1982: 43-61.

R. Carroll. Adequacy in interpretative Sociology, SR, núm. 3, 1982: $392-406$.

Antoine Casanova, Michel Dion. Catbolicisme, bourgeoisie, socialisme, fea dalité et histoire des idéologies, LP, núm. 227, 1982: 12-18.

Jordi Castellanos. Modernisme i estetocràcia, R, núm. 12, 1982: 117-136.

Christie Davies. Etbnic Jokes, moral values and social boundaries, BJS, núm. 3, 1982: 383.403.

Jean Duparc. Une mutation ideologique dans les années trente: à propos de Cbristopher Caudwell, IP, núm. 225, 1982: 25.39.

Terry Eagleton. Wittgenstein's Friends, NLR, núm. 135, 1982: 64-90.

Shamuel N. Eisenstadt. The axial age: the emergence of transcendental visions and the rise of clerics, AES, núm. 2, 1982: 294-314. 
V. Enguelgatdt. La ciencia, la técnica y el bumanismo, CiSo, núm. 1, 1982: 43-55.

Manuel Fernández del Riesgo. Razón técnica y alienación en la sociedad post-industrial, CRS, núms. 20-21, 1982: 87.112.

Peter Fuller. Art and Biology, NLR, nim. 132, 1982: 83-96.

Eric J. Hobsbawn. Home i dona a la iconografia socialista, L'A, núm. 45, 1982: 47.57.

John Hund. Are social facts real?, BJS, núm. 2, 1982: 270-278.

Christian Lalive d'Epinay. Persistance de la culture populaire dans les sociétés industrielles avancées, RFS, núm. 1, 1982: 87-109.

Manuel Maceiras. Actualidad y Prospectiva: exigencias éticas de la contemporaneidad, CRS, núms. 20-21, 1982: 67-86.

Charles Malamoud. Sémantique et rbétorique dans la bierarcbie des «buts de l'bome», AES, núm. 2, 1982: 215-238.

Hussein Mroné. La place du patrimoine islamique dans la pensée contemporaine, LP, núm. 229, 1982: 51-61.

Benjamin Oltra. La restitución de la perspectiva: acerca de la ideología, REIS, núm. 18, 1982: 51-57.

Else Øyen. The Social Functions of Confidentiality, CS, núm. 2, 1982: 1-37.

Jean Claude Passeron, Fernando Porto y François de Singly. Los silencios: contribución a la interpretación de las no-respuestas en las encuestas de opinión, REIS, núm. 17, 1982: 83-136.

Emile Poulat. Le discuors sur les droits de l'bomme: ses paradoxes et ses contraintes, LP, núm. 229, 1982: 18-26.

Jaques Texier. Le privilège épistemologique du présent et la nécessité du moment génétique dans les Grundrisse de K. Marx, LP, núm. 225, 1982: 40.52 .

H. Thoben. Mechanistical and Organistic Analogies in Economics Recon. sidered, KYKLOS, vol. 35, fasc. 2, 1982: 292-306.

Jesús María Vázquez. Apuntes para una Sociología y Deontología del tenómeno televisivo, CRS, núms. 20-21, 1982: 11-66.

Paul Veyne. The inventory of differences, $\mathrm{E} \& \mathrm{~S}, 1982:$ 173-198.

Andrew Wallace-addrill. The Golden Age and Sin in Augustan Ideology, P\&P, núm. 95, 1982: 19-36.

Eviatar Zerubabel. The Standardization of time: A Sociobistorical Perspective, AJS, núm. 1, 1982: 1.23.

Eviatar Zerubabel. Calendars and Group Identity, ASR, núm. 2, 1982: 284-289.

\section{Monogràfics:}

Dossier: Une antbropologie généralisée, CIS, 1982:

Rémi Cliquet. Narcissisme et anomie, 197-222.

David Le Breton. Corps et symbolique sociale, 223-232. 
«Papers»: Revista de Sociologia

Gérard Labounoux. La parodie, instrument du pouvoir symbolique, CIS, 233-250.

Iona Andreco-Miereanu. Espace et temps de la magie dans un village roumain actuel, 251-266.

Gerald Berthoud, Mondher Kilani. Adaptation et resistance-essai de relecture des cultes du cargo, CIS, núm. D, 1982: 267-292.

Marc Le Pape et Claudine Vidal. Raisons pratiques africaines, 293-322.

André Béfin. Le sang, le sens et le travail: Georges Vacber de Laponge, darwinisme social, fondateur de l'antbropologie, 323-344.

Jean-Louis Siran. Genèse des identités de classes et consentement à la domination, 345-362.

Dossier: Rites et fétiches, ARSS, núm. 43, 1982:

Maria Andrea Loyola. Cure des corps et cure des âmes. Les rapports entre les médecines et les religions dans la banlieue de Rio, 3-45.

Martine Dumont. A propos d'une expérience féticbiste de l'écbec scolaire à Brazzaville, 47-57.

Pierre Bourdieu. Les rites d'institution, 58-63.

Fabrizio Sabelli. Le rite d'institution, résistance et domination, 64-69.

Moshé Lewin. Aux rites avec le Stalinisme. Quelques réflexions bistoriques, 71-82.

Dossier sobre Individualité, biographie, société, LP, núm. 228, 1982:

Antoine Casanova. Individualité, biograpbie, société. Quelques problemes et perspectives de recberches, 29-43.

Danielle Blutrach, Alain Chem. Y a-t-il une nouvelle personalité fémenine?, 44-57.

François Hurstel. Identité de père et classe ouvriére a Monttéliard aujourd'bui, 58-72.

Jean-Pierre Cotten. La notion d'individu social dans les «Groundrisse» de Marx, 73-82.

Daniel Becquemont. Travail social, argent et individualité (s) à l'èpoque victorienne, 83-96.

Albert Soboul. Napoléon, le grand bomme et l'bistoire, 97-103.

Jacques Legrand. Type et modele bistorique d'individualité, Ciuggis gan, LP, núm. 228, 1982: 104-115.

Bernard Muldworf. Biograpbie, psycbanalyse, folie. Inconscient et rapports sociaux, $116-129$.

Dossier: El grup de Bloomsbury, L'A, núm. 46, 1982:

Marta Pessarrodona. El grup de Bloomsbury: una aproximacio, $20-22$.

Joaquim Clotet. G. E. Moore o la bumanitat a la càtedra, 23-25.

Marta Pessarrodona. La literatura al Grup de Bloomsbury, 26-29.

Quentin Bell. L'art i l'estètica de Bloomsbury, 30-31.

Francesc de Carreras. Etica i politica a Bloomsbury, 32-35. 
Josep M. Bricall. Jobn Maynard Keynes o el pensament econòmic a Bloomsbury, 36-38.

\section{SOCIOLOGIA ECONOMICA I DEL DESENVOLUPAMENT}

Timothy and Beth Bates. The Desintegration of Poland's Economy, MR, núm, 9, 1982: 11-19.

François Blanchard. Puissance et déclin des Etats-Unis dans les années quatre- vingts, LP, núm. 225, 1982: 13-24.

Alain Booth. Corporatism, Capitalism and deppresion in twentieth century Britain, BJS, núm. 2, 1982: 200-223.

Samuel Cohn. Hetcher's Theory of Regional Underdevelopment, ASR, númeto 4, 1982: 477-488.

Kevin Danaher. Namibia: Profits, Racism, and the Soviet Threat, MR, núm. 8, 1982: 36-47.

Salvador Giner: Political Economy, legitimation and the State in Soutbern Europe, BJS, núm. 2, 1982: 172-198.

Patrick Minford: The New Cambridge Economic Policy: a Critique of its Prescriptions, GO, núm. 1, 1982: 48-60.

Trevor Noble. Recent Sociology, Capitalism and the coming crisis, BJS, núm. 2, 1982: 238-253.

Michael R. Smith. Accounting for inflation in Britain, BJS, núm. 3, 1982: 301-329.

Anthony Winson. The "Prussian Road» of agrarian development, E\&S, núm. 4, 1982: 381-408.

Glenn Yago and Kathleen Blee. The Political Economy of Exploitation A Revision, IS, núm. 2, 1982: 63-72.

\section{SOCIOLOGIA DE L'EDUCACIO}

Jesús Beltrán Llera. El modelado de la conducta social, CRS, núms. 20-21, 1982: 115-140.

M. S. H. Hickox. The Marxist Sociology of Education: A Critique, BJS, nímero 4, 1982: 563-578.

Paul di Maggio. Cultural Capital and School Success, ASR, núm. 2, 1982: 189-201.

Kevin McCormick. Elite Ideologies and Manipulation in Higber Education: the development of a political perspective on bigher education in England, SR, núm. 1, 1982: 45-70.

Daniel Monteux. Enseignement supérieur: ouvrir les voies non balisées du changement, LP, núm. 229, 1982: 4-17. 
«Papers»: Revista de Sociologia

Monogràfics:

Dossier: Classements scolaires et classement social, ARSS, núm. 42, 1982:

Chistopher Charle. Naissance d'un grand corps. L'Inspection des finances à la fin du XIXe. siècle, 3-17.

Annie Verger. L'artiste saisi par l'Ecole. Classements scolaires et «voca" tion》 artistique, 19-32.

Alain Guillemin. Aristocrates, propriétaires et diplômés. La lutte pour le pouvouir local dans le département de la Mancbe, 1830-1875, 33.60.

Marcos Zafiropoulas i Patrice Pinell. Drogues, déclassement et stratégies de disqualification, 61.75 .

Yves Aguilat. La chartreuse de Mirande. Le monument bistorique, produit d'un classement de classe, 76-85.

\section{SOCIOLOGIA DE LA FAMILIA, DELS GRUPS D'EDAT}

I DE LA SEXUALITAT

Y. Michal Bodemann. Class rule as Patronage: Kinship, Local Ruling Cliques and the State in Rural Sardinia, JPS, núm. 2, 1982: 147-175.

José Castillo Castillo. Los bijos de la sociedad de consumo española, REIS, núm. 17, 1982: 39-51.

Yves Clot. Jeunesse, travail, société: voies et enjeux d'une mutation, LP, núm. 225, 1982: 3-12.

Christie Davies. Sexual Taboos and Social Boundaries, AJS, núm. 5, 1982: 1.032-1.064.

Carlos Martínez Shaw. La família, l'infant i la mort. Conversa amb Pbilippe Ariès, L'A, núm. 53, 1982: 65-70.

Jean Kellerhals et Pierre-Yves Troutot. Divorce et modèles matrimoniaux. Quelques figures pour une analyse des regles de léchange, RFS, núm. 2, 1982: 195.222.

Monogràtics:

Dossier: Sexualités occidentales, C, núm. 35, 1982:

Articles de: Philippe Ariès, André Béjin, Michel Foucault, Paul Veyne, Jean-Louis Flandrin (entre d'altres).

Número monogràfic sobre el tema La liberalisation de l'avortement, RFS, núm. 3, 1982. 


\section{SOCIOLOGIA JURIDICA I CRIMINAL}

Francisco Alvira Martín y María Angeles Rubio Rodríguez. Victimización $e$ inseguridad: la perspectiva de las encuestas de victimización en España, REIS, núm. 18, 1982: 29-50.

Lynn McDonald. Theory and evidence of rising crime in the nineteenth century, BJS, núm. 3, 1982: 404-420.

David P. Phillips. The Impact of Fictional Television stories on U.S. Adult Fatalities: New Evidence on the Effect of the Mass Media on Violence, AJE, núm. 6, 1982: 1.340-1.359.

\section{SOCIOLOGIA DE LA MEDICINA}

Bryan S. Tumer. The government of the body: medical regimens and the rationalization of diet, BJS, núm. 2, 1982: 254-269.

Valeria Wassiliewski. Medical Planning for Nuclear War, MR, núm. 8, 1982: 19-27.

\section{SOCIOLOGIA POLITICA}

Richard D. Alba and Cwen Moore. Etbnicity in the American Elite, ASR, núm. 3, 1982: 373-382.

Raymond Aron. Alternation in Government in the Industrialitzed Countries, GO, núm. 1, 1982: 3-21.

Association of Salvadoran Women. Participation of Latin American Women in Social and Political Organizations: Reflections of Salvadoran Women, MR, núm. 2, 1982: 1-24.

Miquel Barceló. L'Islam entre l'imperialisme i el fonamentalisme religiós (1914-1980), L'A, núm. 54, 1982: 22-29.

Anthony Batnett. War over the Falklands, número monogràfic, NLR, número $134,1982$.

Helena Béjar Merino. Evolución del libro político en España: 1970-80, REP, núm. 25, 1982: 151-165.

Luis Beltrán. La teoria budja del poder político. Una concepción tradicio nal africana del poder, REIS, núm. 18, 1982: 59-67.

Gene H. Bell-Villada: Two Americas, Two Woldviews, and a Widening Gap, MR, núm. 5, 1982: 37-44.

Menno Boldt. Intellectual orientations and nationalism among leaders in an internal colony: a theoretical and comparative perspective, $\mathrm{BJS}$, número 4, 1982: 484-510. 
«Papers»: Revista de Sociologia

Roger Burbach. Central America: The end of U.S. Hegemony?, MR, numero 8,1982 : 1-18.

Shirley Cereseto. Socialism, Capitalism and Inequality, IS, núm. 2, 1982: 5-29.

Jacques Couland. Islam et politique. Essai d approcbe, LP, núm. 229, 1982: 41.50 .

Kevin Featherstone. Elections and Parties in Greece, GO, núm. 2, 1982: $180-194$.

Keit Hope. A Liberal Tbeory of Prestige, AJS, núm. 5, 1982: 1.011-1.032.

David Hunt. Village Culture and the Vietnamese Revolution, P\&P, numero 94, 1982: 131-157.

George Katsiaficas. The Extraparlamentary Left in Europe, MR, núm. 4, 1982: 31-45.

Michel Kazin. Conversa amb Edward P. Tbompson. El desarmament nuclear a Europa, L'A, núm. 46, 1982: 60-67.

Arthur Lewin. The Fall of Michael Manley: A Case Study of the Failure of Reform Socialism, MR, núm. 9, 1982; 49-60.

L. J. Macfarlane, Marxist Theory and Human Rigbts, GO, núm. 4, 1982: 414-428.

V. R. Mehta. Centre and Peripbery in Indian Politics, GO, núm. 2, 1982: 164-179.

J. G. Merquier. More Order Than Progress? the Politics of Brazilian Positivism, GO, núm. 4, 1982: 454-468.

István Mészàros. Il rinnovamento del marxismo e l'attualità storica dell' ofensiva socialista, PS, núm. 23, 1982: 5-142.

Maxine Molyneux: Socialist Societies old and New: Progress Toward Women's Emancipation?, MR, núm. 3, 1982: 56-100.

Roger Morgan. Social Democracy in Europe: A Comparative Examination, GO, núm. 1, 1982: 23-34.

Pelai Pagès. Els moviments nacionalistes contemporanis, L'A, núm. 54, 1982: 56-61.

J. E. Pilat. Democracy or Discontent? Ecologists in the European Electoral Arena, GO, núm. 2, 1982: 222-233.

Biancamaria Scarcia Amoretti. Pour une analyse politique de l'economie islamique, LP, núm. 229, 1982: 62-70.

J. B. Skemp. The Causes of Decadence in Plato's Republic, GO, núm. 1, 1982: 80-93.

Nicholas Stacey. Portrait of the Enterpreneur as a Young Man, GO, numero 4, 1982: 444-453.

J. Thomas. The Dominican Model, MR, num. 10, 1982: 34-43.

Sami Zubaida. The ideological conditions for Kbomeini's doctrine of government, E\&S, núm. 2, 1982: 138-172. 
Donald Capps. The Case of John Henry Newman and bis Mentors, JSSR, núm. 1, 1982: 58-70.

Leo Driedger. Individual Freedom versus Community Control, JSSR, número 3, 1982: 226-241.

Louis Dupré. Some Recent Pbilosopbical Discussions of Religion, JHI, núm. 3, 1982: 505-518.

F. F. Fernández. La religiosidad de los universitarios residentes en los colegios mayores de Madrid, CRS, núms. 20-21, 1982: 231-255.

Renée C. Fox. Is Religion Important in Belgitum?, AES, núm. 1, 1982; 3-38.

Santos González López, Sociología del becho religioso. El concepto de secularización: entre el paradigma explicativo y el mito sociologico, CRS, núms. 20-21, 1982: 199-230.

Michael P. Hornsby-Smith et al. A Typology of Englisb Catholics, SR, núm. 3, 1982: 433-459.

James Davison Hunter. Subjectivization and the New Evangelical Theodicy, JSSR, núm. 1, 1982: 39-47.

Ghita Ionescu. Speaking Notes: with the Bisbop of London on How and Why the Church Might Be Losing its Nerve and its role in the Modern Society, GO, núm. 3, 1982: 351-361.

Stephen A. Kent. Relative deprivation and resource mobilization: a study of early Quakerism, BJS, núm. 4, 1982: 529-544.

Lawrence H. Mamiya. From Black Muslim to Bilalian: The Evolution of a Movement, ISSR, núm. 2, 1982: 138-153.

Cburch and Revolution in Nicaragua: An interview with Peter Marcbetti, MR, núm. 3, 1982: 43.55.

Carl Marzani. The Vatican as a left Ally?, MR, núm. 3, 1982: 1-42.

Ian McAllister. The Devil, miracles and the afterlife: The political Socio logy of religion in Northern Ireland, BJS, núm. 3, 1982: 330-347.

W. Alex Mclutosch and Jon P. Alston. Lenski revisited: The Linkage Role of Religion in Primary and Secondary Groups, AJS, núm. 4, 1982: $852-882$.

David A. Snow, Richard Machaleck. On the Presumed Fragility of Unconventional Beliefs, JSSR, núm. 1, 1982: 15-25.

\section{Monografics:}

Dossier extraordinari: Eternelle Eglise?, ARSS, núm. 43, 1982:

Pierre Bourdieu, Monique de Saint Martin. La sainte famille. L'épiscopat français dans le champ du pouvoir, 2.53.

André Rousseau. Le jeune clergé des années 70, 69 .

André Rousseau. L'Action catholique ouvrière, 70-71. 
«Papers»: Revista de Sociologia

Chatles Suand. Conversions religieuses et reconvertions économiques, 72-94.

François Bouvin. L'école catbolique est-elle encore religieuse?, 95-108.

Número monogràfic sobre el tema: $A$ propos de la théorie du charisme, $\mathrm{SC}$, núm. 1, 1982.

Número monogràfc sobre el tema: La pieté populaire dans les régions du nord, de l'est et du centre de l'Europe, SC, núm. 2, 1982.

\section{SOCIOLOGIA RURAL I URBANA}

Georges Augustius. Esquisse d'une comparaison des systemes de perpéturation des groupes domestigues dans les sociétés paysannes européennes, AES, núm. 1, 1982: 39-69.

Judith R. Blau and Peter M. Blau. Metropolitan Structure and Violent Crime, ASR, núm. 1, 1982: 114-128.

Philippe Bourgeois. What U.S. Foreing Policy aces in Rural El Salvador: An Eyewitness Account, MR, núm. 1, 1982: 14-32.

José Cazorła Pérez. Algunos efectos socio-políticos de la inmigración rural en las relaciones interurbanas, REIS, núm. 19, 1982: 105-118.

Jean Claude Combessie. Marché du travail et dynamique des valeurs: la coueillette du coton en Andalousie, ARSS, núm. 41, 1982: 73-85.

Ruth Glass. Divided and Degraded: The Downtrodden Peoples of India, MR, núm. 3, 1982: 101-127.

Enrique Luque Baena. Las Hurdes: apuntes para un análisis antropológico, REIS, núm. 17, 1982: 7-37.

Fred Magdoff. Pros and Cons of Agricultural Mecbanization in the Third World, MR, núm. 1, 1982: 33-46.

Jean Peneff. Carrières et trajectoires sociales des patrons algériens, ARSS, núm. 41, 1982: 61-72.

Placide Rambaud. Les agriculteurs polonais à la conquête de leur identité, ARSS, núm. 41, 1982: 47.59.

José Manuel Sánchez-Díez. Factores sociológicos y bumanos de las explotaciones familiares de ovino en Tierra de Campos, REAS, núm. 118, 1982: 69-123.

Theodor Shanin. Defining Peasants: Conceptualisations and De-Conceptualisations, SR, núm. 3, 1982: 407-432.

Monogràfics:

Monogràfic sobre Le travail social et le local, ES, núm. 40, 1982.

Entre altres articles destaquen: 
Revista de revistes

Alain Bihr. La part maudite da capitalisme, 15-30.

Maurice Imbert. Le travail Social et le local, 31.38.

Jeanne Homina1 et Jacques Ion. Le sociologue urbain, le travailleur social et le local, 39-46.

Alain Bourdin et Núria Puig. Travail social et nouvelles dynamiques loca. les, 57.70 .

Daniel Bachet. Violence, space et babitat, 79-86.

Jean Pierre Garnier. Des inconnus dans la ville, 87-102.

Número monogràfic: Agrarian Movements in India, JPS, núm. 3, 1982.

Número monogràfic sobre sociologia regional i urbana: Article:

Elisabeth Lebas. Urban and Regional Sociology in Advanced Industrial So cieties: A Decade of Marxist and Critical Perspectives, CS, núm. 1, 1982: 1-108.

\section{SOCIOLOGIA DEL TREBALL I DE LES ORGANITZACIONS}

Ferruccio Andolfi. L'utopia del lavoro come bisogno vitale, PS, núm. 23, 1982: $171-189$.

Jacques Aunequin. M. I. Finley et l'esclavage antique, LP, núm. 227, 1982: 36-47.

James N. Baron and William T. Bielby. Technical Relation in the Workplace, ASR, núm. 2, 1982: 175-188.

Harry Braverman. The Degradation of Work in the Twentieth Century, MR, núm. 1, 1982: 1-3.

Deborah Bernstein and Shlmomo Swirsky. The rapid economic development of Israel and the emergence of the etbnic division of labour, BJS, núm. 1, 1982: 64-85.

Stephan Feuchtwang. Occupational ghettos, E\&S, núm. 3, 1982: 251-291. William Finley. Worker's Control and Control of Workers, IS, núm. 2, 1982: 51-62.

Enest Gellnet. The Industrial Division of Labour and National Cultures, GO, núm. 3, 1982: 259-267.

Stuart Henry. The Working Unemployed, SR, núm. 3, 1982: 460-477.

Emmanuele Invernizzi. Politica Sociale. Strategia sindicale, realtà politica e lavorativa di quadri, impiegati e operai, QRS, núm. 95, 1982: 167. 189.

Robert L. Kaufman and Seymour Spilerman. The Age Structures of Ocupations and Jobs, AJS, núm. 4, 1982: 827-851.

J. Miller McPherson and L. Smith-Lovin. Women and Weak Ties: Differences by ex in the Size of Voluntary organizations, AJS, num. 4, 883.904 . 
«Papers»: Revista de Sociologin

Michael Poole. Tbeories of industrial democracy, SR, núm. 2, 1982: 181207.

Michael Waillace and Atne L. Kalleberg. Industrial transformation and the Destine of Craft, ASR, núm. 3, 1982: 307-324.

Monogràfics:

Número monogràfic sobre: Il funzionamiento del sindicato, QRS, núm. 94, 1982.

Número monogràfic sobre el tema: Trabajo, Salud Ergonomía, ST, números $7-8,1982$. 